\title{
Cuestionario para la clasificación de consumidores de cigarrillo (C4) para jóvenes*
}

\section{Questionnaire to Classify the Level of Tobacco Consumption Young People}

\author{
Constanza Londoño Pérez** \\ Universidad Católica de Colombia \\ Ivonne Rodríguez Rodríguez \\ Universidad Católica de Colombia \\ Carlos Andrés Gantiva Díaz \\ Universidad Católica de Colombia \\ Recibido: 31 de enero de 2011 \\ Revisado: 2 de mayo de 2011 \\ Aceptado: 30 de junio de 2011
}

\section{Resumen}

Este estudio tuvo como objetivo el diseño y validación de una escala para la clasificación de adolescentes y jóvenes consumidores de tabaco, de acuerdo con la frecuencia e intensidad de consumo y problemas asociados, intención de cesación y signos de dependencia nicotínica. La muestra estuvo conformada por 448 universitarios de ambos sexos con edades entre los 18 y 31 años, provenientes de cuatro instituciones educativas ubicadas en tres ciudades. El análisis psicométrico mostró que la prueba cuenta con la confiabilidad y validez necesarias para ser utilizada en poblaciones similares. De acuerdo con los resultados las conclusiones se centran en la amplia posibilidad de uso del cuestionario y las bondades de un sistema de clasificación de consumo tabáquico no centrado exclusivamente en la frecuencia y cantidad de cigarrillos fumados.

Palabras clave: consumo de tabaco, dependencia, validez y confiabilidad.

\section{Abstract}

This study had as primary target the design and validation of one scale that allowed the classification of 
the consuming university tobacco adolescents, in agreement with frequency, intensity, and problems associated with the consumption, intention of cessation and the presence of signs of nicotine dependency. The sample was conformed of 448 university students, both sorts to an age between the 18 and 31 years; originate of four located educative institutions in three cities. The advanced psychometric analysis showed that the test count the conditions of trustworthiness and validity that are necessary to be used in populations similar of validation. The conclusions were concentrate in the plenty possibility of using of the questionnaire and kindness to exclusively no count on a system of classification of tobacco consumption in the frequency and amount of smoked cigarettes.

Keywords: tobacco consumption, dependency, validity and confusability.

\section{Introducción}

Las sustancias psicoactivas legales son las de mayor consumo entre los adolescentes de América Latina según la Organización Panamericana de la Salud (OPS, 2006), en especial se ha hecho cada vez más frecuente el consumo de tabaco. En Colombia de acuerdo con los informes existentes al menos el $18 \%$ de jóvenes entre 13 y 18 años consumió tabaco alguna vez en la vida (Encuesta Mundial de Tabaquismo, 2001; Encuesta Mundial sobre el consumo de tabaco y jóvenes, 2000) y casi el $20 \%$ de personas entre los 18 y 19 años son fumadores actuales (Instituto Nacional de Cancerología, 2006; Ariza \& Nebot, 2002), todo a pesar de que la OMS ya ha identificado la existencia de una estrecha relación entre fumar y un sin número de enfermedades crónicas altamente prevenibles que son causa cada año de al menos el $5 \%$ del total de muertes en el mundo (OMS, 2006; Becoña \& Miguez, 2004).

Los adolescentes han sido identificados como uno de los grupos de más alto riesgo de tabaquismo debido a que el inicio es cada vez a más temprana edad y los reportes anuales de consumo muestran un incremento anual del $5 \%$ en el número de fumadores adolescentes (Encuesta mundial sobre tabaquismo en jóvenes de Bogotá, 2001; Barrueco, Hernández \& Torrecilla, 2003) especialmente en el ingreso a la universidad (Organización Panamericana de la Salud, 2001), esto soporta la necesidad de desarrollar investigaciones que aborden el problema de consumo desde el diagnóstico hasta el desarrollo de programas de prevención y tratamiento.

\section{Evaluación del consumo de cigarrillo}

Según la OMS (2006) el tipo de consumo está asociado con el número de cigarrillos consumidos al día, así que se ha desarrollado como estrategia para evaluar el nivel de consumo asumiendo como factor principal la frecuencia diaria de consumo, sin tener en cuenta otros aspectos clave como la intensidad de la ingesta y los problemas asociados al consumo. Así de acuerdo con la OMS (2003) los fumadores se clasifican en leves, moderados y severos en la siguiente escala:

- Fumador leve: consume menos de 5 cigarrillos diarios.

- Fumador moderado: fuma un promedio de 6 a 15 cigarrillos diarios.

- Fumador severo: fuma más de 16 cigarrillos por día en promedio.

Existe otro tipo de fumadores que no encajan en esta clasificación debido a que tienen un nivel de consumo bajo pero constante, denominados Chipping o fumadores ligth. Este tipo de fumadores parece estar protegido ante la adicción, pues cuentan con un grupo de factores protectores como con una alta valoración al éxito académico, locus de control interno, soporte social y bajo consumo de cigarrillo entre padres y amigos (Presson, Chassin \& Sherman, 2002). Este es un ejemplo de las dificultades existentes para establecer una clasificación única que incluya a todo tipo de fumadores; por eso cada autor ha agrupado y denominado a los fumadores de forma distinta de acuerdo con criterios particulares para 
definir el nivel de consumo. En el caso de los chipping en algunos estudios son llamados fumadores sociales, chippers o indulgentes y en otros se consideran como diferentes las tres clasificaciones (Shiffman \& Jean, 2006) sin explicar las razones para definir los parámetros usados en la misma.

En esta línea, se ha clasificado a los fumadores de acuerdo con el nivel de dependencia a la nicotina, a través de la Escala Fagerstrom (Becoña, 2003) que diferencia a los fumadores blandos que no tienen niveles altos de consumo, de los fumadores duros que presentan uso compulsivo del tabaco (Becoña \& Lorenzo, 2004); esta escala considera a la nicotina como el principal factor reforzante del consumo tabáquico, restándole importancia a factores psicológicos y sociales asociados al proceso de adicción al tabaco. En la misma línea se encuentra la Escala del Síndrome de Dependencia de la Nicotina (NDSS) elaborada por Becoña, Fernández, López y Míguez (2009) usada para identificar dependencia a la sustancia en personas que buscan ayuda para dejar de fumar; al igual que la escala Fagerstrom clasifica en dos grandes grupos a los fumadores los dependientes y los blandos; pero sin duda aún personas que fuman a diario pueden no presentar síntomas de abstinencia ante la reducción o abandono del consumo (Campo, 2005) e incluso no haber experimentado problemas de salud asociados al consumo.

Por ello la evaluación y clasificación de los niveles de consumo ha sido una de las principales dificultades en el estudio del consumo de esta sustancia, ya que no existen más que cuatro o cinco escalas o entrevistas básicas de consumo que no facilitan la ubicación respecto del riesgo mayor o menor de los fumadores. Estos instrumentos de evaluación son usados por profesionales de la salud en el proceso de tamizaje y se reducen a dos o tres preguntas referidas al conocimiento de los efectos nocivos del cigarrillo, al deseo de abandonar el consumo y a los intentos anteriores por dejar de consumir y acerca de la recurrencia o recaídas presentadas (Campo, 2005); pero no abordan aspectos como la intensidad del consumo (concentración nicotínica y profundidad de la inhalación), la frecuencia o los problemas asociados.
Entre ellos se encuentra la Entrevista de Hábitos en Relación con la Nicotina de Bejerot, Knorring y Ekeselius (2001), en la que se indaga el consumo diario, el uso de parches y chicles con nicotina; y se plantea que el uso mantenido de un cigarrillo al día durante seis meses implica tabaquismo. De otra parte, la Entrevista de Evaluación del Consumo de Tabaco propuesta por Becoña, Míguez, López, Vázquez Lorenzo (2006) incluye varias preguntas relacionadas con, si alguna vez en la vida la persona ha fumado, si ha fumado en los últimos seis meses y la cantidad de cigarrillos consumida.

El Cuestionario de consumo de tabaco diseñado por Archila, García y Londoño (2005) es un registro de frecuencia e intensidad de consumo que incluye, además, la descripción de los lugares en los que se fuma y el respeto o no de las normas y leyes que regulan el consumo en ciertos espacios. Adicionalmente, existen dos sistemas internacionales de clasificación de la dependencia nicotíni$\mathrm{ca}$, el CIE - 10 y el DSM en los que se define que para ser considerada dependiente una persona debe cumplir al menos cuatro de los síntomas definidos; sin que se planteen indicadores para la clasificación de fumadores no dependientes.

Adicionalmente, Lujic, Reuter y Netter (2005) han propuesto una clasificación de los motivos más frecuentes que las personas reportan para consumir tabaco, pero no proponen algún instrumento para medirlos.

También, Lira, González, Medina, Cruz y Vega (2009) diseñaron un cuestionario para evaluar las situaciones relacionadas con el consumo que influyen en el proceso de dejar de fumar y la abstinencia prolongada, usado, principalmente, para determinar los motivadores del consumo; sin que se haga ninguna clasificación específica respecto del nivel de consumo.

En suma, los cuestionarios, entrevistas y escalas disponibles no permiten diferenciar de manera sensible los tipos de consumo existentes, que van más allá de una simple división dicotómica entre duros y blandos, o dependientes y no dependientes; ya que existen fumadores de distintos tipos y grados de consumo, es preciso diseñar escalas 
que permitan alcanzar tal meta para ofrecer atención específica según cada tipo de consumo, hacer tamizajes para detección oportuna de casos, plantear programas de prevención del consumo de tabaco en poblaciones similares a la incluida en el presente estudio, adelantar estudios comparativos de consumo por poblaciones y evaluar los alcances reales de las distintas acciones centradas en la prevención del consumo de nicotina. En busca de resolver estas dificultades para clasificar a los fumadores, en el grupo de investigación en Psicología de la Salud y en el Centro de Estudios e Investigaciones sobre Adicciones y Violencia (CEIAV) de la Universidad Católica de Colombia, se ha desarrollado un instrumento para clasificar efectivamente a los consumidores de tabaco.

\section{Método}

\section{Participantes}

La muestra no probabilística estratificada de acuerdo con la carrera, el semestre y las instituciones de procedencia estuvo conformada por 448 estudiantes universitarios que correspondían al $20 \%$ del total de los estudiantes matriculados en la institución, de los cuales 160 eran mujeres y 64 hombres (con una omisión de respuesta acerca del género), cuyas edades oscilaban entre los 18 y los 31 años con una media de 22,7 años y desviación estándar de 3.21 años, es decir, que la muestra incluyó tanto adolescentes como jóvenes.

Entre los criterios de inclusión se encontraban: tener entre 18 años cumplidos y 30 años, la expresión del deseo de participar en la investigación y la firma del consentimiento informado, estar matriculado en una de las 4 instituciones incluidas en la investigación.

\section{Instrumento}

El cuestionario para la Clasificación de Consumidores de Cigarrillo (C4) que mide el nivel de consumo de cigarrillo en estudiantes universitarios, se diseñó a partir de las clasificaciones existentes y se retomaron algunos aspectos clave de otros cuestionarios, entre los que están el
Test de Fagerström de dependencia a la nicotina (Heatherton, 1991 adaptación española de Becoña, 1994) de donde se retoman la lista de señales de dependencia a la nicotina y el cuestionario de Consumo de Tabaco (Archila, García \& Londoño, 2005) del que se retoman dos preguntas acerca de la intención de dejar de fumar y los intentos previos; además se incluyen otras categorías complementarias como la intensidad con la que se ingiere la nicotina (concentración por cigarrillo y profundidad de la inhalación), los problemas asociados al consumo, la frecuencia de consumo y otros aspectos relacionados con el consumo en estudiantes universitarios. Se sugiere aclarar que su uso debería apoyarse en pruebas fisiológicas que permitan reconfirmar el autorreporte dado por las personas que lo contestan.

No se usan escalas alternas para la validación concurrente debido a la no existencia de una prueba que evalúe exactamente el mismo constructo que la escala validada en el presente estudio, por ello, deben conducirse nuevos estudios para contrastar los resultados obtenidos.

\section{Procedimiento}

Fase 1. Definición del constructo, nivel de consumo de acuerdo con los estándares generales de la OMS (2006): señales de dependencia, intensidad, frecuencia y problemas asociados al consumo de cigarrillo. Además de la revisión de las escalas, cuestionarios y entrevistas existentes, para definir aspectos relevantes que debían ser considerados en el cuestionario que se pretendía desarrollar.

Fase 2. Diseño de los ítems y las opciones de respuesta, un grupo conformado por un médico, un magíster en adicciones y violencia, un magíster en psicología de la salud y una psicóloga. El proceso de diseño incluyó la extracción de ítems claves de los inventarios antes mencionados.

Fase 3. Evaluación por jurados, una vez diseñado el cuestionario se procedió a someterlo a la evaluación de 3 jurados expertos, quienes analizaron el instrumento en general, respecto de la validez de contenido, la suficiencia, pertinencia y redac- 
ción de los ítems y de las opciones de respuesta. Se analizó el nivel de concordancia entre los jurados y se incorporaron las sugerencias realizadas por estos.

Fase 4. Pilotaje y aplicación, se realizó un pilotaje preliminar mediante la aplicación del cuestionario a 20 jóvenes para evaluar la presentación del cuestionario; posteriormente se llevó a cabo la aplicación del instrumento para determinar sus características psicométricas a la población elegida. La aplicación del instrumento fue realizada por uno de los investigadores a estudiantes de diversos semestres y carreras, quienes firmaron el consentimiento. El cuestionario fue aplicado de forma colectiva en grupos por semestres intactos o de conveniencia en espacios cedidos por los docentes, cuya aplicación tuvo un tiempo promedio de 30 minutos. Únicamente 3 participantes excedieron el límite de los 30 años, por ello no fueron excluidos de la muestra.

Fase 5. Elaboración de la base de datos y análisis estadísticos, se adelantaron análisis descriptivos y de las condiciones psicométricas del instrumento, usando el paquete estadístico SPSS 15, para determinar validez, confiabilidad y comportamiento de cada uno de los ítems. Para todos los ítems con opciones de respuesta distintas se uso el índice de consistencia interna Alpha de Cronbach, pues aunque el error puede incrementarse en la inclusión de ítems dicótomos, el C4 constituye una sola prueba y no es recomendable darle un tratamiento como si fueran escalas independientes, tal como lo anota Pardo, Rocha, Avendaño y Barrera (2005).

\section{Resultados}

Los conceptos emitidos por los tres jurados expertos a quienes se les envió el cuestionario mostraron un alto nivel de concordancia (92\%) respecto de la importancia de tener en cuenta las variables incluidas en la clasificación del nivel de consumo de tabaco en adolescentes y jóvenes universitarios.

\section{Análisis de las condiciones psicométricas de la prueba}

De acuerdo con los análisis psicométricos adelantados, la prueba cuenta con un nivel alto de confiabilidad ya que el Alpha de Cronbach reportado para la escala fue de 0.90 . En el análisis por ítem se muestra que todos aportan a la confiabilidad general de la prueba y por tanto, se decide mantener el instrumento con los ítems originales ( $\mathrm{Ta}$ bla 1).

Tabla 1.

Análisis de la confiabilidad por ítems

\begin{tabular}{ccc}
\hline Ítem & $\begin{array}{c}\text { Correlación con } \\
\text { el puntaje total }\end{array}$ & $\begin{array}{c}\text { Alpha si el } \\
\text { item es borrado }\end{array}$ \\
\hline 1 &, 7541 &, 9086 \\
2 &, 8503 &, 9070 \\
3 &, 9017 &, 9023 \\
4 &, 8689 &, 9043 \\
5 &, 6843 &, 9054 \\
6 &, 9340 &, 8944 \\
7 &, 2455 &, 9160 \\
8 &, 8430 &, 9070 \\
9 &, 6927 &, 9060 \\
10 &, 9291 &, 8942 \\
11 &, 8172 &, 9079 \\
12 &, 7451 &, 9097 \\
13 &, 3932 &, 9147 \\
14 &, 5368 &, 9132 \\
15 &, 6972 &, 9039 \\
\hline
\end{tabular}

\section{Calificación}

Posterior a la normalización de los puntajes se identificaron los puntos de corte de cada nivel teniendo en cuenta la desviación estándar; así, la calificación de cada factor se obtuvo al dar una puntuación entre 0 y 6 dependiendo del nivel de riesgo que representaba cada condición evaluada, siendo 0 el menor riesgo y 6 el más alto riesgo. El puntaje máximo posible era de 50 y significaba que la persona tenía la más fuerte dependencia a la nicotina y el puntaje mínimo era 0 y se daba cuando la persona no fumaba actualmente o nunca había probado el cigarrillo. La escala de clasificación iba de 0 a 50 , y se dividió en cuatro niveles de consumo: bajo (1 a 5 puntos), modera- 
do (6 - 17 puntos), alto con señales de inicio de dependencia (18 a 29 puntos) y dependiente (30 a 50); puntuación que se definió de acuerdo con el cumplimiento de diversos criterios de intensidad, frecuencia, problemas asociados y presencia de indicadores de dependencia nicotínica.

\section{Consumo de tabaco}

El 54\% de los estudiantes reportan no fumar actualmente o nunca haber fumado, del grupo total de fumadores se encuentra que la mayor parte de ellos presentan señales de dependencia nicotínica o un nivel alto de consumo, 128 y 56 correspondientemente. El consumo moderado y bajo son los menos frecuentes, 16 y 8 para cada caso. No se encontraron diferencias de consumo respecto del sexo, aun cuando la muestra estudiada estaba conformada predominantemente por mujeres (Tabla 2).

Tabla 2.

Clasificación según niveles de consumo y género

\begin{tabular}{lccccccccc}
\hline & \multicolumn{1}{c}{ Totales } & \multicolumn{2}{c}{ Total } & \multicolumn{2}{c}{$M$} & \multicolumn{2}{c}{$F$} \\
Consumo & $\mathbf{f}$ & $\%$ & $\begin{array}{c}\text { Nivel } \\
\text { consumo }\end{array}$ & $\mathbf{f}$ & $\%$ & $\mathbf{f}$ & $\%$ & $\mathbf{f}$ & $\%$ \\
$\begin{array}{l}\text { No } \\
\text { fumadores }\end{array}$ & 240 & 54 & Leve & 8 & 3 & 4 & 5 & 4 & 3 \\
Fumadores & 208 & 46 & Moderados & 16 & 6 & 6 & 7 & 10 & 8 \\
Total & 448 & & Alto & 76 & 31 & 16 & 20 & 40 & 32 \\
& & & Dependie. & 148 & 60 & 56 & 68 & 72 & 57 \\
& & Total & 208 & 100 & 82 & 100 & 126 & 100 \\
\hline
\end{tabular}

\section{Discusión}

De acuerdo con los resultados obtenidos es posible concluir que la prueba cuenta con un alto nivel de confiabilidad y validez de constructo, esto puede deberse a que el diseño del instrumento se hizo a partir del desarrollo de nuevos ítems y la integración de preguntas incluidas en otras pruebas previamente validadas, y a que la propuesta surge de una amplia y profunda revisión acerca de las variables asociadas con los distintos tipos de consumo. Se conservan los 14 ítems propuestos originalmente teniendo en cuenta que todos aportan a la confiabilidad general de la prueba y corresponden a diferentes dimensiones del consumo de cigarrillo, dimensiones que son considera- das indispensables para alcanzar una clasificación global y efectiva del nivel de consumo que incluye frecuencia, intensidad, problemas asociados y síntomas psicológicos y físicos de dependencia.

Respecto de la clasificación propuesta por la OMS (2003) en la que se plantea la existencia de tres niveles de consumo leve, moderado y severo, se reafirma la idea de que la clasificación no puede ser hecha tomando como único punto de referencia el número de cigarrillos, ya que no todos los jóvenes que reportaron tener mayor frecuencia de consumo habían presentado señales de dependencia nicotínica o experimentado el craving.

Al comparar el cuestionario de Clasificación del Consumo de Cigarrillo C4 con otras opciones de evaluación se evidencia que este cuestionario es congruente con la clasificación sugerida por la OMS en la que el tipo de consumo ofrece varias categorías: leve, moderado y severo; pero contiene además, el nivel de dependencia que sobrepasa el consumo duro o riesgoso, incluyendo síntomas de abstinencia y otros aspectos clave, como intensidad de la ingesta y problemas asociados al consumo. Igualmente, permite identificar fumadores ligth que tienen un consumo constante pero bajo, que de acuerdo con la propuesta de Presson, Chassin y Sherman (2002) y de Shiffman y Jean (2006) deben ser identificados y estudiados, ya que parecen tener una cierta invulnerabilidad hacia la adicción y su consumo es motivado casi exclusivamente en situaciones de intercambio social.

De otra parte, al incluir los ítems de dependencia de la Escala Fagerström y el Cuestionario de consumo de tabaco se facilita tanto la discriminación de los adictos (Heatherton, 1991 adaptación española de Becoña, 1994; Becoña \& Lorenzo, 2004) como la descripción detallada de la topografía del consumo en términos de intensidad, frecuencia y situaciones de consumo (Archila, García \& Londoño, 2005), que se incluyen respectivamente en la Escala del Síndrome de Dependencia de la Nicotina (NDSS) (Becoña, Fernández, López y Míguez, 2009) y en la Entrevista de Evaluación del Consumo de Tabaco (Becoña, Míguez, López, Vázquez \& Lorenzo, 2006). Además engloba otros as- 
pectos psicológicos y sociales que no son incluidos en estas escalas como el deseo de abandonar el consumo, los intentos previos de cesar el consumo acerca de la recurrencia y recaídas presentadas, que son importantes en cualquier proceso de cambio iniciado (Londoño, 2007; Campo, 2005).

Aunque el C4 incluye aspectos relevantes relacionados con la absorción de la nicotina y la profundidad de la inhalación del humo reportada y el grado de control del consumo en situaciones de prohibición legal o médica reportado en la prueba, este cuestionario no indaga acerca de los motivos para el consumo, y las situaciones que funcionan como disparadores que se investigan en el cuestionario de Consumo de Lira, González, Medina, Cruz y Vega (2009); no obstante, estos aspectos hacen parte más de la dimensión general del consumo, mantenimiento o abandono de la práctica, por tanto su uso puede ser complementario en procesos de acompañamiento para cesación de consumo de cigarrillo. La estandarización y validación del $\mathrm{C} 4$ en otras poblaciones facilitara la definición de una clasificación más universal y única que incluya a todo tipo de fumadores, facilitando así el diagnóstico adecuado a partir de criterios específicos por nivel de consumo y la comunicación entre profesionales de la salud.

En suma, los cuestionarios, entrevistas y escalas disponibles no permitían diferenciar de manera más sensible los tipos de consumo existentes, que van más allá de una simple división dicotómica entre duros y blandos, o dependientes y no dependientes; ya que existen fumadores de distintos tipos y grados de consumo es preciso diseñar escalas que faciliten alcanzar tal meta para ofrecer atención específica y diferencial según cada tipo de consumo. Finalmente, en concordancia con Becoña (2007; 2009), es cada vez más evidente la importancia de contar con un instrumento que incluya aspectos psicológicos y sociales del consumo de tabaco que facilitan la comprensión de las razones del consumo y de los patrones comportamentales asociados a este; sumados al efecto reforzante que tiene la nicotina en sí misma

Respecto del consumo de tabaco y en oposición con otros estudios (Informe de Resultados de la
Encuesta Mundial de Tabaquismo realizada en Bogotá, 2001; Sistema Interamericano de Datos Uniforme sobre Consumo de Drogas, 2003; OPS, 2001, 2003). Las cifras muestran que casi la mitad de los estudiantes universitarios de la muestra son fumadores, con un grupo importante de jóvenes con consumo alto y dependiente, en la misma línea estudios como el de Archila, García y Londoño (2005), el de Caballero, González, Pinilla y Barber (2005), el de la Organización Panamericana de la Salud (OPS, 2003) y el de Bolzan y Peleteiro (2003) ya habían hecho notar el grave problema de consumo tabáquico en los jóvenes.

\section{Limitaciones}

Ya que los motivos del consumo pueden ser tomados como un indicador sería deseable incluir algunos ítems que permitieran evaluar este aspecto y así dar mayor alcance a la prueba en la clasificación precisa del tipo de consumo de quien la responde, ya que la motivación es uno de los principales componentes de la dimensión psicológica de la prevención, tal como lo plantean Flórez (2007), Londoño y Vinaccia (2005) y Londoño (2007).

Aunque la prueba alcanzó un buen nivel de confiabilidad se sugiere adelantar estudios epidemiológicos que faciliten la confirmación de los hallazgos obtenidos en el presente estudio, usando entrevistas a manera de Gold Estándar que faciliten contrastar la validez concurrente del C4.

\section{Referencias}

Archila, D., García, D. \& Londoño, C. (2005). Estrategias de afrontamiento y consumo de cigarrillo en estudiantes de la Universidad Santo Tomás. Experiencias Investigativas, 2, 9-27.

Ariza, C. \& Nebot, M. (2002). La prevención del tabaquismo en los jóvenes: realidades y retos para el futuro. Monografía Tabaco, 16(2).

Barrueco, M., Hernández, M. \& Torrecilla, M. (2003). Manual de prevención y tratamiento del tabaquismo. Madrid, España: Ergon. 
Becoña, E. \& Lorenzo, M.C. (2004). Evaluación de la conducta de fumar. Monografía tabáquica, 16(2), 201-226.

Becoña, E. \& Miguez, M. (2004). Consumo de tabaco y psicopatología asociada. Psicooncología, 1(1), 99-112.

Becoña, E. (2007). Bases psicológicas de la prevención del consumo de drogas. Papeles del Psicólogo, 28(1), 11-20.

Becoña, E., Fernández, H., López, A. \& Míguez, M.C. (2009). La escala del Síndrome de Dependencia de la Nicotina (NDSS) en una muestra de fumadores que demandan tratamiento para dejar de fumar. Psicothema, 21(4), 579-584.

Becoña, E., Míguez, M.C., López, A., Vázquez, M.J. \& Lorenzo, M.C. (2006). Resiliencia y consumo de tabaco en jóvenes. Prevención de Tabaco, 8(3), 98-107.

Bolzan, A. \& Peleteiro, R. (2003). Tabaquismo durante la adolescencia temprana. Estudio en escolares argentinos. Journal de Pediatria, 79(5), 461-466.

Bejerot, S., Knorring, S. \& Ekselius, L. (2001). Rasgos de personalidad y tabaquismo en pacientes con trastorno obsesivo-compulsivo. European Psychiatry, 8, 11-18.

Caballero, A., González, B., Pinilla, J. \& Barber, P. (2005). Factores predictores del inicio y consolidación del consumo de tabaco en adolescentes. Gaceta Sanitaria, 19(6), 440-447.

Encuesta mundial sobre el consumo de tabaco y jóvenes. (2000). El consumo de tabaco entre los jóvenes: informe de vigilancia. Bulletin of the World Health Organization, 78(7), 868-876.

Encuesta mundial sobre el tabaquismo en jóvenes de Bogotá. (2001). Organización Mundial de la Salud. Bogotá, Colombia.
Flórez, L. (2007). Psicología Social de la Salud Promoción y Prevención. Bogotá, Colombia: Manual Moderno.

Lira, J., González, F., Medina, V., Cruz, S. \& Vega, C. (2009). Análisis psicométrico del inventario situacional para consumidores de tabaco. Diversitas: Perspectiva en Psicología, 5(1), 65-76.

Londoño, C. (2007). Construcción del modelo cognitivo social integrado para la prevención del consumo de alcohol en adolescentes universitarios. Típica,3(2). Boletín Electrónico de Salud Escolar. http/www.tipica.org.co.

Londoño, C. \& Vinaccia, S. (2005). Prevención del abuso en el consumo de alcohol en jóvenes universitarios: Lineamientos en el diseño de programas costo-efectivos. Psicología y Salud, 15(2), 241-249

Lujic, C., Reuter, M.; Netter, P. (2005). Psychobiological theories of smoking and smoking motivation. European Psychologist, 10(1), 1-24.

Organización Mundial de la Salud. (2003). www. who.int Extraído en mayo de 2006.

Organización Mundial de la Salud. (2006). Informe Nacional de Salud. "Colaboremos por la salud”. París, Francia.

Organización Panamericana de la Salud. (2003). Por una juventud sin tabaco. Adquisición de habilidades para una vida saludable. Washington, USA.

Organización Panamericana de la Salud. (2003). Tomado de la página de la OPS 2006. www. ops.int

Organización Panamericana de la Salud. (2006). "Encuesta Latinoamericana de Consumo de Drogas”. Washington, USA.

Pardo, C., Rocha, M., Avendaño, B. \& Barrera, L. (2005). Manual de procesamiento de datos y análisis de ítems para el segundo estudio regional comparativo y explicativo. LLECE. UNESCO. 
Presson, C., Chassin, L. \& Sherman, S. (2002). Psychosocial antecedents of tobacco chipping. Health Psychology, 20(4), 384-392.

Shiffman, S. \& Paty, J. (2006). Smoking patterns and dependence: contrasting chippers and heavy smokers. Journal of Abnormal Psychology, 115(3), 509-523.
Sistema Interamericano de Datos Uniformes sobre Consumo de Drogas. (2003). Estudio comparativo del consumo de drogas. Manual de Prevención, Módulo 3, Naciones Unidas, Oficina Contra la Droga y el Delito. 


\section{CUESTIONARIO PARA LA CLASIFICACIÓN DE CONSUMIDORES DE CIGARRILLO (C4) (Versión estudiantes universitarios Londoño, Constanza y Rodríguez, Ivonne 2006)}

Género:

Semestre:

1. ¿Actualmente fuma?

sí

Edad: Universidad:

2. ¿Ha fumado por más de seis meses alguna vez en su vida?

Sí Carrera:

3. ¿Hace cuánto tiempo empezó a fumar?
a. Menos de un año
d. Entre 3 y 5 años
b. Un año
e. Más de 5 años
c. Más de un año
f. Más de 10 años

4. ¿Cuántos cigarrillos fuma en un día normal de consumo?
a. 10 o menos
c. 21 a 30 cigarrillos
b. 11 a 20 cigarrillos
d. 31 o más

5. ¿Cuánto tarda, después de despertarse en fumar su primer cigarrillo?
a. Menos de 5 minutos
c. Entre 31 minutos y 1 hora
b. Entre 6 y 10 minutos
d. Más de una hora

6. ¿Cómo fuma los cigarrillos?
a. Traga el humo
b. Tiene el humo en la boca

7. ¿Desearía dejar de fumar?

8. ¿Ha intentado dejar de fumar?

$$
\text { sí }
$$

sí

9. ¿Fumar le ha acarreado problemas de salud? sí
NO

NO

NO

10. Cuando deja de fumar un cigarrillo habitual, experimenta: (puede marcar el número de opciones que crea necesario)
a. Irritabilidad o ira
d. Dolor de cabeza
b. Impaciencia
e. Tensión o ansiedad
c. Dificultad para concentrarse
f. Somnolencia

11. ¿Alguien de su familia le ha pedido que deje de fumar?

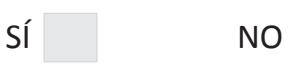

12. ¿Algún profesional de la salud le ha sugerido que deje de fumar?

NO

13. ¿Encuentra difícil dejar de fumar en sitios donde está prohibido?

NO

14. ¿Encuentra difícil dejar de fumar aun cuando está enfermo?

sí

NO

15. ¿A qué cigarrillo odiaría más renunciar?
a. Al primero de la mañana
d. Al de después del almuerzo
b. Al que acompaña un trago
e. Al último de la noche
c. Al de antes de un parcial 


\section{CUESTIONARIO PARA LA CLASIFICACIÓN DE CONSUMIDORES DE CIGARRILLO (C4)}

(Constanza Londoño Pérez 2006 - Clave de Clasificación)

Género:

Semestre:

1. ¿Actualmente fuma?

\begin{abstract}
Sí
\end{abstract}
(1)

NO

(0)
Carrera:

2. ¿Ha fumado por más de seis meses alguna vez en su vida? Sí

(1) NO

(0)

3. ¿Hace cuánto tiempo empezó a fumar?
a. Menos de un año (1)
d. Entre 3 y 5 años (4)
b. Un año (2)
e. Más de 5 años (5)
c. Más de un año (3)
f. Más de 10 años (6)

4. ¿Cuántos cigarrillos fuma en un día normal de consumo?
a. 10 o menos (1)
c. 21 a 30 cigarrillos (3)
b. 11 a 20 cigarrillos (2)
d. 31 o más (4)

5. ¿Cuánto tarda, después de despertarse en fumar su primer cigarrillo?
a. Menos de 5 minutos (4)
c. Entre 31 minutos y 1 hora (2)
b. Entre 6 y 10 minutos (3)
d. Más de una hora (1)

6. ¿Cómo fuma los cigarrillos?

a. Traga el humo (4)

b. Tiene el humo en la boca (2)

7. ¿Desearía dejar de fumar?

8. ¿Ha intentado dejar de fumar?

9. ¿Fumar le ha acarreado problemas de salud? sí

\section{sí}

sí No

$\mathrm{NO}$
NO

¿Cuántas veces?

10. Cuando deja de fumar un cigarrillo habitual, experimenta: (puede marcar el número de opciones que crea necesario)
a. Irritabilidad o ira
d. Dolor de cabeza
g. Ninguna de las anteriores
b. Impaciencia
e. Tensión o ansiedad
c. Dificultad para concentrarse
f. Somnolencia

11. ¿Alguien de su familia le ha pedido que deje de fumar?

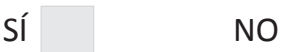

12. ¿Algún profesional de la salud le ha sugerido que deje de fumar?

sí

NO
NO
NO
NO

13. ¿Encuentra difícil dejar de fumar en sitios donde está prohibido?

sí

NO
NO
NO
NO

14. ¿Encuentra difícil dejar de fumar aun cuando está enfermo?

Sí

NO
NO
NO
NO

15. ¿A qué cigarrillo odiaría más renunciar?
a. Al primero de la mañana (4)
d. Al de después del almuerzo (2)
b. Al que acompaña un trago (1)
e. Al último de la noche (3)
c. Al de antes de un parcial (3) 